\title{
Low-Back Pain and Knee Position-Related Differences on Postural Control Measures During a One-Legged Stance in Athletes
}

\author{
Alexandre H. Nowotny, Mariane Guizeline Calderon, Bruno Mazziotti O. Alves, Marcio R. de Oliveira, \\ Rodrigo A. de Carvalho Andraus, Andreo F. Aguiar, Cesar F. Amorim, Guillaume Leonard, \\ and Rubens A. da Silva
}

\begin{abstract}
Context: Chronic low-back pain (CLBP) may be associated with changes in postural balance in athletes as poor postural control during sports practice. Objective: To compare the postural control of athletes with and without CLBP during 2 one-legged stance tasks and identify the center of pressure (COP) cutoff values to determine the main differences. Designed: A cross-sectional study. Setting: Laboratory of functional evaluation and human motor performance. Participants: A total of 56 male athletes, 28 with and 28 without CLBP (mean age $=26$ y). Intervention: The one-legged stance with knee extension and with the knee at $30^{\circ}$ flexion tasks were measured and analyzed on a force platform. The participants completed three 30-second trials $(30 \mathrm{~s}$ of rest between each trial). Main Outcome Measures: The COP parameters: the area of COP, mean COP sway velocity in both the anteroposterior and mediolateral directions, and total COP displacement were computed, and a receiver operating characteristics curve analysis was applied to determine the group differences. Results: Athletes with CLBP had poorer postural control $(P<.01)$ in both tasks. The $30^{\circ}$ knee flexion reported more postural instability than the knee extension for all COP parameters (a large effect size $d=0.80$ ). The knee extension cutoffs identified were $>7.1 \mathrm{~cm}^{2}$ for the COP area, $>2.6 \mathrm{~cm} / \mathrm{s}$ for the COP sway velocity in the anterior-posterior direction, and $>3.2 \mathrm{~cm} / \mathrm{s}$ for the mediolateral direction. Whereas, the $30^{\circ}$ knee flexion cutoffs were $>10.9$ $\mathrm{cm}^{2}$ for the COP area, $>2.9 \mathrm{~cm} / \mathrm{s}$ for the COP sway velocity in the anterior-posterior direction, and $>4.1 \mathrm{~cm} / \mathrm{s}$ for the mediolateral direction. Both measures showed enough sensitivity and specificity (ie, area under the curve $=0.88$ in and 0.80 , respectively) to discriminate both groups. Conclusions: The athletes with CLBP had poorer postural control than the healthy athletes and obtained specific cutoff scores from the COP values.
\end{abstract}

Keywords: posture, spine, chronic low-back pain, athletes, postural balance

The prevalence of chronic low-back pain (CLBP) is high $(61 \%)$ in sports, which is not so far from the $85 \%$ found in the general population. ${ }^{1}$ The CLBP represents a $30 \%$ incidence in sports and $10 \%$ to $15 \%$ of total sports injuries, with high treatment costs. ${ }^{2}$ This musculoskeletal disorder limits physical performance and can cause athletes to drop out of training and competition. ${ }^{1,3}$

The majority of low-back injuries in athletes occur during competitions and are often diagnosed as nonspecific contusions, sprains, or strains, which may be defined as pain, muscle soreness, or discomfort in the lumbar and sacral regions, with or without referral pain to the buttocks or legs, which has been present for more than 1 week and/or has interrupted at least one training

\footnotetext{
Nowotny, Calderon, Oliveira, Andraus, Aguiar, and da Silva are with the Rehabilitation Sciences UEL/UNOPAR, LAFUP-UNOPAR, Londrina-PR, Brazil. Amorim and da Silva are with the Département des Sciences de la Santé, Programme de physiothérapie de l'Université McGill offert en extension à l'Université du Québec à Chicoutimi Saguenay, Chicoutimi, QC, Canada. da Silva is also with the Département des Sciences de la Santé, Clinique Universitaire de physiothérapie (CUpht), Centre intersectoriel en santé durable, UQAC, Lab BioNR, CUpht, UQAC, Université du Québec à Chicoutimi, Saguenay, Québec, Canada. Alves and Amorim are with the Department of Physical Therapy, University of the City of São Paulo, UNICID, São Paulo, Brazil. Alves is also with the Paris Saint Germain Department of Physical Therapy and Biomechanics, Paris, France. Amorim is with the Department of Physical Therapy - Human Performance Laboratory at the College of Nursing \& Health Sciences, Florida International University, Miami, FL, USA. Leonard is with the School of Rehabilitation, Faculty of Medicine and Health Sciences, Research Center on Aging, Institute of Geriatrics, Université de Sherbrooke, Sherbrooke, QC, Canada. da Silva (rubens.dasilva@uqac.ca) is corresponding author.
}

session. ${ }^{1,4}$ Several sport postures require maintaining the body in an upright standing position for long periods of time, especially to perform sports-related gestures (tennis, volleyball, and basketball involving jumps; taekwondo; and soccer), which could increase the risk of developing CLBP. ${ }^{3}$ It must be noted that inadequate lumbar stability could potentially explain pain and disability. ${ }^{5-8}$ In fact, trunk muscle fatigue can increase neuromuscular deficits, resulting in brief uncontrolled intervertebral movements and, consequently, lumbar spine instability and back pain. ${ }^{6-8}$ This phenomenon, although reported in nonathlete adults, could be generalized to athletes, as muscle fatigue and postural imbalance in sports actions could theoretically overload the spine, resulting in pain. ${ }^{9,10}$

Some evidences suggests that CLBP may be associated to changes in postural balance in athletes. ${ }^{2,11}$ However, few studies have reported specific results on the deficits of the mechanisms of postural control in athletes with CLBP. ${ }^{3,6,8}$ Summarizing the literature in general on individuals with CLBP, trunk postural control has been shown to be impaired while standing upright on 2 legs $^{3,12,13}$ or in a one-legged stance, ${ }^{3,14-16}$ as well as when sitting in a challenging, unstable "wobbling" chair. ${ }^{17,18}$ Most challenging balance conditions can further discriminate better balance deficits than simple tests on CLBP comparisons (ie, CLBP poorer than healthy control), ${ }^{15}$ which is also in line with the conclusions reached by a recent review on this issue. ${ }^{19} \mathrm{~A}$ brief explanation for poor balance in general CLBP people from the facet of spine instability concept could be associated with changes in trunk muscular pattern, ${ }^{20,21}$ back muscle fatigue, ${ }^{6,22}$ and lower proprioception. ${ }^{10,23}$ This could also be in line for athletes. 
Our research team was thus interested in investigating the onelegged balance stance, which is considered a more challenging task to assess postural control in athletes with and without CLBP. In fact, the one-legged stance with knee extension has been examined in previous studies, including those athletes with CLBP. 7,14,24-27 However, knee extension constitutes a locking mechanism, a kinematic phenomenon also known as the screw home that increases knee stability ${ }^{28}$; contrary, the knee flexion position above $20^{\circ}$ constitutes a position frequently adopted during most sports where the screw home mechanism is absent. At this knee position, the individual can present an articular mobility with signs of joint instability $^{28}$ and, consequently, increase their chances to have lumbar or lower-limb instability and back injury. ${ }^{29}$ From this perspective, one research question arises: Can the one-legged stance with $30^{\circ}$ knee flexion position be a determinant and a discriminative condition for postural control assessment in athletes with CLBP?

Furthermore, the literature offers limited data of cutoff rates related to postural control parameter associated to the center of pressure (COP) measures on a force platform for the one-legged stance to determine the differences on the threshold of balance deficits in individuals with CLBP when compared with healthy control. Therefore, the main purpose of this study was, first, to compare 2 knee positions during a one-legged stance, extension (EXT) and $30^{\circ}$ knee flexion (FLEX $30^{\circ}$ ), in athletes with and without CLBP, and second, to determine the COP sway cutoff scores from a receiver operating characteristics (ROC) curve to truly identify athletes with CLBP based on poor postural control. Our hypothesis was that athletes with CLBP would present greater postural instability for both tasks, although FLEX $30^{\circ}$ would be more sensitive and discriminative than EXT to better report these differences on COP sway cutoff scores.

\section{Methods}

\section{Design}

This study was a cross-sectional design, including a case-control group of athletes without CLBP. This study was approved by the UNOPAR University Ethics Committee (protocol number: 1860811). No incentives or financial benefits were offered to take part in the study.

\section{Participants}

A total of $56(n=56)$ male athletes (aged between 18 and $40 y)$ were recruited from the sports centers and randomized into 2 groups: a group with CLBP and GWCLBP (without CLBP). Athlete recruitment was done through flyers, newspapers, radio, and television. All participants were informed of the procedures, risks, and benefits of the investigation and signed an approved informed consent.

The athletes with CLBP were defined as being in daily or almost daily pain for a minimum of 3 months. The pain location was described by the participants as being between the flotation ribs and the posterior margin of the iliac crest and sacrum. Participation in level-I (jumping, pivoting, hard cutting, like in basketball, handball, and soccer) or level-II sports (less jumping or hard cutting than level I, like in volleyball, racket sports, and martial arts). ${ }^{30}$ Athletes should be training, playing, or both at least 4 times a week. ${ }^{30}$ The athletes were at the elite, college high division, professional, or Olympic level. The participants were currently competing in some official federation or confederation event (national or international). ${ }^{31}$
The following exclusion criteria were considered: previous musculoskeletal surgery, any kind of musculoskeletal disorder excepting CLBP, neurological disorder, and visual problems. ${ }^{25}$ The use of corrective contact lenses was permitted.

We used data from a previous study to estimate the sample size needed to identify the differences in postural control measures between the athletes with and without CLBP. ${ }^{3}$ Based on the mean values of the COP sway displacement, during a bipedal stance test with eyes open, of healthy participants $(2.7[0.79] \mathrm{mm})$ and those with CLBP (3.5 [1.0] mm/s), 18 participants would be needed per group to run an unpaired $t$ test $(95 \% \mathrm{CI})$ with a power of 0.80 .

\section{Procedures}

The data collection was completed in one 2-hour session. All procedures were carried out in the morning and afternoon in a quiet laboratory room with controlled indoor temperature $\left(22^{\circ} \mathrm{C}\right)$ and light intensity. The same investigator (trained physiotherapist) performed all the procedures with the participants to ensure uniformity.

All participants were given 2 minutes of familiarization time on the force platform before the tests began. The participants received oral instructions about standard positioning and postural alignment during all procedures.

Pain intensity was perceived just before starting the test with a $10-\mathrm{cm}$ visual analog scale and the following score range: $0=$ no pain and $10=$ worst pain. The pain quality was assessed with the Short Form of the McGill Pain Questionnaire, which contains 15 descriptors of pain sensation, with each descriptor ranked on a 4-point rating scale: 0 , none; 1 , mild; 2 , moderate; and 3, severe. The Pain Rating Index is composed of the sum of all 15 descriptors with the total score ranging from 0 to 45 . Perception of disability with the Oswestry Disability Index, including ten 6-point scales (0-5; total 50 points), the total Oswestry Disability Index score ranging from $0 \%$ (no disability) to $100 \%$ (maximum disability), and the sum of the 10 Oswestry Disability Index scores is expressed as a percentage of the maximum scores. The Fear-Avoidance Beliefs Questionnaire (FABQ) was used to evaluate whether athletes with CLBP were afraid and believed that their physical activity affected their low back pain. The FABQ is defined by a score range of 0 to 42 for both conditions (physical activity and work); the higher scores represent greater fear and beliefs.

For balance measurement, the vertical ground reaction signal forces data from the force platform (EMG System Ltda; São Paulo, SP, Brazil) were sampled at $100 \mathrm{~Hz}$ and filtered with a 35-Hz low-pass, second-order Butterworth filter, and afterward, it was converted into COP data using MATLAB-based routines (MathWorks, Natick, MA). The main COP parameters computed were as follows: (1) $95 \%$ confidence elliptical area of COP (in $\mathrm{cm}^{2}$ ) and (2) mean COP sway velocity (VEL in $\mathrm{cm} / \mathrm{s}$ ) in both the anteroposterior (VEL A/P in $\mathrm{cm} / \mathrm{s}$ ) and mediolateral (VEL M/L) directions and the total COP displacement (TCD in $\mathrm{cm}){ }^{4,15}$ These parameters were calculated for the total duration of each trial and the mean of the 3 trials were analyzed. ${ }^{32}$

The participants were standing on their preferred leg for EXT and the FLEX $30^{\circ}$, with the contralateral knee flexed at $90^{\circ}$, the arms kept parallel to the trunk, with the eyes open. ${ }^{32}$ During the FLEX $30^{\circ}$, the standing leg was maintained at $30^{\circ}$ of knee flexion. ${ }^{32}$ The FLEX $30^{\circ}$ was measured manually with a universal goniometer (WCS; Cardiomed, Espírito Santo, Brazil) ${ }^{32}$ and controlled by verbal feedback from the evaluator through clinical visual analysis during testing. The balance conditions were presented randomly (dark plastic bag containing 2 papers labeled EXT and FLEX $30^{\circ}$ ). 
Foot positioning was standardized using a tape marker on the force platform surface. The participants were instructed to maintain their body position during the tasks looking at a target (black cross $=14.5$ height $\times 14.5$ width $\times 4 \mathrm{~cm}$ thickness) attached to the wall at eye level with a frontal distance of $2 \mathrm{~m} .{ }^{32}$ The participants performed three 30 -second trials for each task, with 30 seconds of rest between them. ${ }^{15,32}$ See the illustration in Figure 1A and $1 \mathrm{~B}$.

\section{Statistical Analyses}

Data normality was confirmed using the Shapiro-Wilk Test. Unpaired $t$ tests were used to compare the anthropometric characteristics, and 2-way analysis of variance was used to compare the groups (group with CLBP and GWCLBP), tasks (EXT and FLEX $30^{\circ}$ ), and interactions. The effect size between groups was calculated using the equation $d=\mathrm{m}_{1}-\mathrm{m}_{2} / \mathrm{SDm}_{2}$, where $\mathrm{m}_{1}$ is the mean of the CLBP group, $\mathrm{m}_{2}$ is the mean of the control group, and $\mathrm{SDm}_{2}$ is the SD of the control group. Based on the Cohen criteria, $d=0.2$ to 0.49 is small, $d=0.5$ to 0.79 is medium, and $d \geq 0.8$ is large. ${ }^{33}$

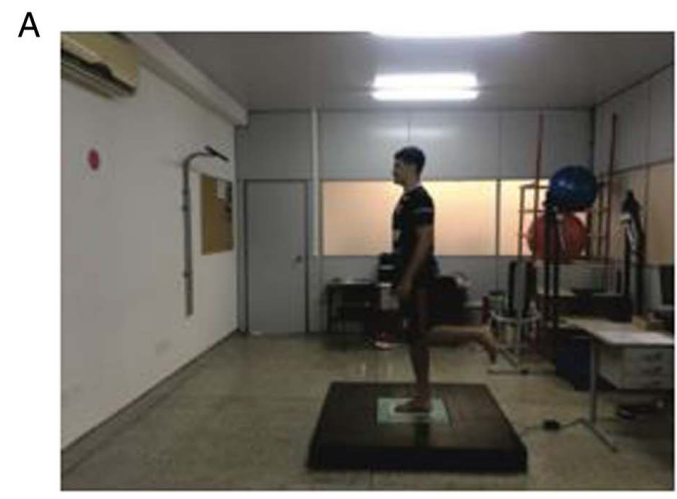

The ROC curve data were used to determine the COP parameter cutoff values related to sensitivity and specificity to identify the participants with CLBP. ${ }^{34}$ The area under the curve (AUC) was used to describe the test's overall performance. The AUC $\geq 0.70$ indicates acceptable discriminatory power, and 0.50 corresponds to random classification. ${ }^{34}$ Statistical analyses were performed using SPSS statistical software (version 24, SPSS Inc; Chicago, IL) and MedCalc (version 17.1; MedCalc Software, Ostend, Belgium) at a .05 significance level.

\section{Results}

Both groups were homogeneous for anthropometric variables, except for height, where the group with CLBP was slightly smaller compared with GWCLBP (Table 1). However, all COP parameters remained unchanged when height was included as a covariate ( $F$ : $0.005-1.84, P=.181-0.942$ ), which supports the subsequent analysis reported in Table 2 .

There was no significant interaction between groups and tasks for postural control measures (Table 2). Significant

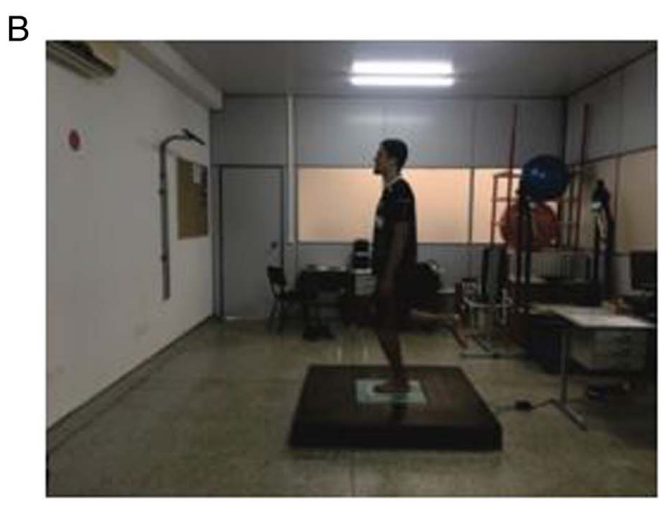

Figure 1 - (A) One-legged stance with knee extension and (B) one-legged stance $30^{\circ}$ knee flexion. Both with eyes open.

Table 1 Participants' Characteristics

\begin{tabular}{|c|c|c|c|}
\hline Variables & GCLBP & GWCLBP & $P$ value \\
\hline Age, y & $25(21-30)$ & $25(22-27)$ & .799 \\
\hline Mass, $\mathrm{kg}$ & $77(73-95)$ & $80(73-93)$ & .774 \\
\hline Height, m & $1.78(1.71-1.85)$ & $1.84(1.76-1.87)$ & $.037 *$ \\
\hline BMI, $\mathrm{kg} / \mathrm{m}^{2}$ & $25(24-28)$ & $24(23-28)$ & .376 \\
\hline Training experience, $y$ & $5(2-7)$ & $5(3-7)$ & .447 \\
\hline Training frequency, $\mathrm{d} / \mathrm{wk}$ & $5(4-5)$ & $5(4-5)$ & .357 \\
\hline Training hours, wk & $10(8-10)$ & $10(8-10)$ & .357 \\
\hline Time since onset of pain symptoms, mo & $36(12-60)$ & l & \\
\hline VAS pain, $\mathrm{cm}$ & $4(3-5)$ & / & \\
\hline ODI & $6(5-9)$ & l & \\
\hline FABQp score & $15(10-20)$ & / & \\
\hline FABQw score & $10(0-15)$ & / & \\
\hline SF-MPQ & $21(11-24)$ & I & \\
\hline
\end{tabular}

Abbreviations: BMI, body mass index; CLBP, chronic low-back pain; FABQp, Fear-Avoidance Beliefs related to physical activity; FABQw, Fear-Avoidance Beliefs related to work; GCLBP, athletes' group with CLBP; GWCLBP, athletes' group without CLBP (control); ODI, Oswestry Disability Index; SF-MPQ, Short form of the McGill Pain Questionnaire; VAS, visual analog scale. Note: Median values with interval interquartile [ii].

*Significant difference between groups $(P<.05)$. 
Table 2 Comparison Between Groups and Tasks

\begin{tabular}{|c|c|c|c|c|c|c|}
\hline \multirow[b]{2}{*}{ Variables } & \multirow[b]{2}{*}{ CLBP } & \multicolumn{2}{|c|}{ Balance tasks } & \multicolumn{3}{|c|}{$P$ value } \\
\hline & & EXT & FLEX $30^{\circ}$ & Group & Tasks & Interaction \\
\hline \multirow[t]{2}{*}{ A-COP, $\mathrm{cm}^{2}$} & CLBP & $9.25(1.9)$ & $11.9(3.7)$ & $<.001 *$ & $<.001 *$ & .147 \\
\hline & WCLBP & $\begin{array}{c}6.15(1.7) \\
d=2.64\end{array}$ & $\begin{array}{c}10.3(3.7) \\
d=0.43\end{array}$ & & & \\
\hline \multirow[t]{2}{*}{ VEL A/P, cm/s } & CLBP & $3.40(1.04)$ & $3.93(1.37)$ & $.009^{*}$ & $.015^{*}$ & .190 \\
\hline & WCLBP & $\begin{array}{c}2.87(0.92) \\
d=0.17\end{array}$ & $\begin{array}{c}3.03(1.07) \\
d=0.84\end{array}$ & & & \\
\hline \multirow[t]{2}{*}{ VEL M/L, cm/s } & CLBP & $4.23(1.09)$ & $4.70(1.14)$ & $<.001 *$ & $.033^{*}$ & .266 \\
\hline & WCLBP & $\begin{array}{c}3.31(1.00) \\
d=0.92\end{array}$ & $\begin{array}{c}3.46(0.85) \\
d=1.45\end{array}$ & & & \\
\hline \multirow[t]{2}{*}{$\mathrm{TCD}, \mathrm{cm}^{2}$} & CLBP & $181(50)$ & $170(46)$ & $.006^{*}$ & .766 & .214 \\
\hline & WCLBP & $\begin{array}{l}144(43) \\
d=0.86\end{array}$ & $\begin{array}{l}151 \\
d=0.46\end{array}$ & & & \\
\hline Mean ES, $d$ & - & 1.14 & 0.80 & & & \\
\hline
\end{tabular}

Abbreviations: ANOVA, analysis of variance; A-COP, area of the COP; CLBP, chronic low-back pain; COP, center of pressure; ES, effect size; EXT, extension; FLEX $30^{\circ}$, $30^{\circ}$ knee flexion; TCD, total COP displacement; VEL A/P, COP velocity in the anteroposterior direction; VEL M/L, COP velocity in the mediolateral direction; WCLBP, without CLBP. Note: Means (SD) of A-COP: 95\% confidence ellipse area of the COP; VEL A/PVEL M/L directions; TCD; mean ES (d); individual balance task effect size parameter $(d)$.

ANOVA results: $* P<.05$-Significant differences of main effects. ${ }^{a}$ Comparison between groups, CLBP (with CLBP) and no WCLBP, for each balance task from EXT and FLEX $30^{\circ}(P<.05$ significant differences between groups).

Table 3 AUC for the Athletes With CLBP, Sensitivity, and Specificity for the Force Platform Variables During EXT and FLEX $30^{\circ}$ Tasks

\begin{tabular}{|c|c|c|c|c|c|c|c|}
\hline Task & Force plate variable & AUC & Sensitivity, \% & Specificity, \% & $95 \% \mathrm{Cl}$ & $P$ & Cutoff \\
\hline EXT & A-COP, $\mathrm{cm}^{2}$ & 0.88 & 93 & 78 & $0.76-0.95$ & $<.001^{*}$ & $>7.1$ \\
\hline EXT & VEL A/P, cm/s & 0.67 & 79 & 57 & $0.53-0.79$ & $<.020 *$ & $>2.6$ \\
\hline EXT & VEL M/L, $\mathrm{cm} / \mathrm{s}$ & 0.74 & 93 & 57 & $0.61-0.85$ & $<.001 *$ & $>3.2$ \\
\hline EXT & $\mathrm{TCD}, \mathrm{cm}$ & 0.76 & 96 & 57 & $0.63-0.86$ & $<.001 *$ & $>127.1$ \\
\hline FLEX $30^{\circ}$ & $\mathrm{A}-\mathrm{COP}, \mathrm{cm}^{2}$ & 0.64 & 68 & 68 & $0.50-0.76$ & $<.062$ & $>10.9$ \\
\hline FLEX $30^{\circ}$ & VEL A/P, cm/s & 0.71 & 71 & 71 & $0.57-0.82$ & $<.002 *$ & $>2.9$ \\
\hline FLEX $30^{\circ}$ & VEL M/L, $\mathrm{cm} / \mathrm{s}$ & 0.80 & 68 & 82 & $0.67-0.90$ & $<.001 *$ & $>4.1$ \\
\hline FLEX $30^{\circ}$ & $\mathrm{TCD}, \mathrm{cm}$ & 0.63 & 85 & 46 & $0.49-0.76$ & $<.069$ & $>131.2$ \\
\hline
\end{tabular}

Abbreviations: AUC, area under the curve; A-COP, area of the COP; CI, confidence interval; CLBP, chronic low-back pain; COP, center of pressure; EXT, extension; FLEX $30^{\circ}, 30^{\circ}$ knee flexion; ROC, receiver operating characteristic, TCD, total COP displacement; VEL A/P, COP velocity in the anteroposterior direction; VEL M/L, COP velocity in the mediolateral direction.

*Statistically significant differences between participants with and without CLBP $(\mathrm{n}=56)$. Knee position: EXT and FLEX $30^{\circ}$.

differences from the analysis of variance were, however, reported for the group and task factors. The GWCLBP showed poorer postural control than control (mean $d=0.96$ ) for all COP parameters, with a strong effect size for the FLEX $30^{\circ}$ task $(d=0.80)$, as well as for the EXT task $(d=1.14)$. In addition, significant differences were found between the 2 knee positions for main COP measurements (area and velocity sway, except for TCD) with more postural instability (greater COP values) being revealed for FLEX than for EXT.

For both knee position tasks, the AUC varied from 0.63 to 0.88 with sensitivity reaching $96 \%$ (Table 3 ). To the EXT, the AUC results were 0.67 to 0.77 , AUC $\geq 0.7$ indicating acceptable discriminatory power to determine the COP parameter cutoffs in athletes with CLBP. In the same way, to the FLEX $30^{\circ}$, only VEL A/P and VEL M/L AUC with $\geq 0.7$ and $\geq 0.8$, respectively, dictated acceptable cutoffs. The most expressive values from AUC sensitivity and specificity were total COP displacement (TCD) with $96 \%$ and VEL M/L FLEX $30^{\circ}$ with $82 \%$. The specific cutoff values detected for the COP parameters that differentiated athletes with CLBP during the EXT task were as follows: $>7.1 \mathrm{~cm}^{2}$ for $95 \%$ confidence elliptical area of COP, $>2.67 \mathrm{~cm} / \mathrm{s}$ for VEL A/P, $>3.26 \mathrm{~cm} / \mathrm{s}$ for VEL M/L, and $>127.1 \mathrm{~cm}^{2}$ for TCD. The results for the FLEX $30^{\circ}$ were $>2.9 \mathrm{~cm} / \mathrm{s}$ for VEL A/P and $>4.1 \mathrm{~cm} / \mathrm{s}$ for VEL M/L.

\section{Discussion}

This study aimed first to compare the postural control on 2 knee positions during a one-legged stance, that is, EXT and FLEX $30^{\circ}$ in athletes with and without CLBP and, second, to determine the COP sway cutoff scores. The findings are in agreement with our hypothesis. Athletes with CLBP presented poorer postural control than healthy controls during the 2 knee positions. Expectedly, the FLEX $30^{\circ}$ position showed more instability than EXT in athletes with CLBP. This study was the first to demonstrate the ROC curves 
and AUC-specific cutoff points for COP variables related to balance deficits in athletes with CLBP during FLEX $30^{\circ}$ and EXT. From ROC curve, AUC measures and main COP parameters (area and velocity) were useful (sensitivity reaching 93\%) for identifying athletes with CLBP related to a healthy control.

\section{Comparison Between Groups}

Our results are in accord with Oyarzo et al, ${ }^{3}$ who reported poor postural balance in 20 athletes with CLBP during a 2-legged stance task compared with 24 healthy athletes. Similarly, AppiahDwomoh et $\mathrm{al}^{24}$ observed significant COP displacements during the one-legged stance in athletes with CLBP when compared to healthy athletes, this condition was close to the ones used in the present study. In addition, during a single-leg squat exercise, Borujeni and Yalfani ${ }^{35}$ reported poor postural control in athletes with CLBP, which in turn, was improved after an 8-week program of respiratory muscle training combined with weightlifting and powerlifting exercises. ${ }^{35}$ Contrarily, Mueller et al, ${ }^{36}$ evaluating COP parameters during the one-legged stance task, reported no differences between athletes with and without back pain.

In general, several results confirm that challenging postural control tasks are better to discriminate those with and without CLBP, as supported previously when postural demands increase, such as a unipodal posture and/or eyes-closed conditions of balance. ${ }^{15}$ Our sample with CLBP reported a strong effect for the FLEX $30^{\circ}$ task $(d=0.80)$, as well as for the EXT task $(d=1.14)$ on group postural control comparisons. The group differences could be explained by various factors affecting balance. In general, for the CLBP population (young and older), poor balance can be linked to changes in trunk-altered muscle coordination, ${ }^{20,21}$ back muscle fatigue, ${ }^{22}$ lower proprioception, ${ }^{23}$ delayed reflex responses, ${ }^{37}$ and anticipatory postural adjustments. ${ }^{38}$ These factors could be related in some way for athletes with CLBP and explain our findings, mainly for athletes from level I and $\mathrm{II}^{30}$ modalities, such as soccer, football, basketball, tennis, judo, karate, and jiujitsu, where the prevalence of back pain is of concern.

In addition, some studies have reported differences in back muscle strength ${ }^{39}$ and endurance (fatigue) ${ }^{6,40}$ in athletes with versus without CLBP. Other studies report differences in motor control impairment, ${ }^{41}$ neuromuscular imbalance, ${ }^{10}$ lower activation of back extensor muscles, ${ }^{6}$ and a delay in the onset latency and lower amplitude of long latency responses (ie, altered reflex responses) in athletes with CLBP. ${ }^{42}$ Again, these differences could, in part, explain our results regarding the influence of these factors on postural control in our samples of athletes.

We also assumed that the differences observed in the present study were due to back-related functional decline (as mentioned above) more than the clinical subjective status of athletes. Trunk motor control deficits in acute and subacute low-back pain are not directly associated with pain or fear of movement. ${ }^{43}$ However, for our chronic sample, pain levels on the day of testing were moderate (mean visual analog scale $=4$ ), and our athletes reported minimal disability and fear of movement. It is possible that our sample of athletes were thus classified more as confronters than avoiders (ie, avoiders would report more fear of [re]injury during the behavioral test, leading to a worse performance) ${ }^{44}$ However, given the cross-sectional nature of our study, it is unclear if trunk functional decline due to back pain led to poor postural control, or if poor postural control resulted in low-back pain. Longitudinal studies are required to further explore this issue in athletes.

\section{Knee Position Differences on Postural Control}

In previous studies, including one from our research team, ${ }^{26}$ the one-legged stance task was a sufficient challenge to discriminate postural instability between different sports modalities and training experience, such as taekwondo, handball, American football, soccer, and nonathletes, ${ }^{14,26,27}$ which supports the use of this condition in the present study. However, no study has investigated the effects of knee posture at $30^{\circ}$ of flexion on postural control based on COP parameters in athletes with CLBP, which in turn, adds a challenge to the discussion of our findings.

However, we found a previous study reporting the reliability of the Biodex Stability System indices using 2 knee positions in the measurement protocol (either permitting a slight knee flexion or maintaining knees locked in total extension) in 21 healthy female adults (mean age 23 y). ${ }^{45}$ Overall, knee flexion was the more reliable condition (intraclass correlation coefficient around .90 for some Biodex indices of stability) compared with knee in total extension. ${ }^{45}$ In addition, the authors reported better balance stability for the flexion position than the extension position, which was contrary when analyzing men with and without CLBP while mainly using the indices from COP parameters, as the present study did. We assumed that, to the level I and II athletes $^{30}$ (eg, soccer, football, tennis, judo) who often adopt FLEX $30^{\circ}$ during competition, FLEX $30^{\circ}$ would be a useful condition to identify individuals at risk of CLBP. The findings of this study support that FLEX $30^{\circ}$ was a more challenging task than EXT to show balance deficits in men athletes with CLBP (higher COP values).

For both tasks of knee position, the AUC varied from 0.63 to 0.88 with sensitivity reaching $96 \%$, which was excellent from a clinical decision-making perspective. The ROC curve and AUC cutoff scores reported here may be a future guide for new studies (prospective) to reach other samples, such as female athletes, and allow early detection of athletes with a potential for balance problems (from a diagnostic perspective). Also, these estimates could be used for longitudinal intervention studies (clinical trials), associating CLBP-related postural control measurement before and after balance rehabilitation or training. At the FLEX $30^{\circ}$ condition, the VEL A/P parameter showed $71 \%$ of sensitivity, while VEL M/L showed $82 \%$ of specificity, suggesting a good accuracy to determine the neuromuscular adjustments during balance performance. Finally, we assumed that our results have implications for health sports professionals to better assess and prescribe specific balance exercises, including the flexed knee position at $30^{\circ}$, to prevent and rehabilitate athletes with CLBP.

\section{Limits of Study}

Some limitations should be reported. The FLEX $30^{\circ}$ was measured with a manual goniometer with verbal feedback from the evaluator during testing to maintain the position for 30 seconds. Nevertheless, all the participants maintained the position during data collection without difficulty. Sitting, lying sports, and swimming were not included to avoid postures that differed from the orthostatic position. The samples were only composed of men. The female pelvic structure, levels of strength, and body composition may influence postural sway ${ }^{46,47}$; thus, these results would be generalized in the future for this sample. In fact, water and sitting sports (eg, cycling, rowing), and female athletes must also be considered in future investigations. 


\section{Conclusions}

Male athletes with CLBP have poorer postural control than those without CLBP, mainly for the $30^{\circ}$ knee flexion balance task. Sensitivity and specificity of the COP parameters were excellent (area and velocity sways), while the cutoff scores from these estimates were useful to classify athletes with CLBP compared with the healthy control. These results have implications for clinical decision making on sports rehabilitation programs to improve balance in athletes with CLBP.

\section{Acknowledgments}

The authors gratefully acknowledge the CAPES (doctoral fellowship) and Global Affairs Canada for a scholarship in the Canadian Program of Future Leaders in America to the first author (A.H.N.). The authors would like to thank the assistance of physical therapy students of the university and laboratory collaborators for their help with the data collection and analysis. The authors declare no conflicts of interest.

\section{References}

1. Trompter K, Fett D, Platen P. Prevalence of back pain in sports: a systematic review of the literature. Sports Med. 2017;47(6):11831207.

2. Mortazavi J, Zebardast J, Mirzashahi B. Low back pain in athletes. Asian J Sports Med. 2015;6(2):e24718. PubMed ID: 26448841 doi:10.5812/asjsm.6(2)2015.24718

3. Oyarzo CA, Villagrán C, Silvestre RE, Carpintero P, Berral FJ. Postural control and low pain in elite athletes comparison of static balance in elite athletes with and without low back pain. J Back Musculoeskelet Rehabil. 2014;27(2):141-146. doi:10.3233/BMR130427

4. Caffaro RR, França FJR, Burke TN, Magalhães MO, Ramos LAV, Marques AP. Postural control in individuals with and without nonspecific chronic low back pain: a preliminary case-control study. Eur Spine J. 2014;23(4):807-813. PubMed ID: 24570125 doi:10.1007/ s00586-014-3243-9

5. Panjabi MM. A hypothesis of chronic back pain: ligament subfailure injuries lead to muscle control dysfunction. Eur Spine J. 2006; 15(5):668-676. doi:10.1007/s00586-005-0925-3

6. Correia JP, Oliveira R, Vaz JR, Silva L, Pezarat-Correia P. Trunk muscle activation, fatigue and low back pain in tennis players. J Sci Med Sport. 2016;19(4):311-316. PubMed ID: 25987492 doi:10. 1016/j.jsams.2015.04.002

7. Granata KP, Gottipati P. Fatigue influences the dynamic stability of the torso. Ergonomics. 2008;51(8):1258-1271. PubMed ID: 18608477 doi:10.1080/00140130802030722

8. Reeves NP, Cholewicki J, Dieën JH, Kawchuk G, Hodges PW. Are stability and instability relevant concepts for back pain? J Orthop Sports Phys Ther. 2019;49(6): 415-424. PubMed ID: 31021689 doi:10.2519/jospt.2019.8144

9. Hrysomallis C. Balance ability and athletic performance. Sports Med. 2011;41(3): 221-232. PubMed ID: 21395364 doi:10.2165/ 11538560-000000000-00000

10. Renkawitz T, Boluki D, Grifka J. The association of low back pain, neuromuscular imbalance, and trunk extension strength in athletes. Spine J. 2006;6(6):673-683. PubMed ID: 17088198 doi:10.1016/j. spinee.2006.03.012

11. Kiers H, Van Dieen J, Dekkers H, Wittink H, Vanhees L. A systematic review of the relationship between physical activities in sports or daily life and postural sway in upright stance. Sports Med. 2013;43(11):1171-1189. PubMed ID: 23955562 doi:10.1007/ s40279-013-0082-5

12. Mazaheri M, Coenen P, Parnianpour M, Kiers H, Dieën JH. Low back pain and postural sway during quiet standing with and without sensory manipulation: a systematic review. Gait Posture. 2013;37(1): 12-22. PubMed ID: 22796243 doi:10.1016/j.gaitpost.2012.06.013

13. Ruhe AR, Fejer R, Walker B. Center of pressure excursion as a measure of balance performance in patients with non-specific low back pain compared to healthy controls: a systematic review of the literature. Eur Spine J. 2011;20(3): 358-368. PubMed ID: 20721676 doi:10.1007/s00586-010-1543-2

14. Matsuda S, Demura S, Uchiyama M. Centre of pressure sway characteristics during static one-legged stance of athletes from different sports. J Sports Sci. 2008;26(7):775-779. PubMed ID: 18409108 doi:10.1080/02640410701824099

15. da Silva RA, Vieira ER, Fernandes KBP, et al. People with chronic low back pain have poorer balance than controls in challenging tasks. Disabil Rehabil. 2018;40(11):1294-1300. doi:10.1080/09638288. 2017.1294627

16. da Silva RA, Vieira ER, Carvalho CE, Oliveira MR, Amorim CF, Neto EN. Age-related differences on low back pain and postural control during one-leg stance: a case-control study. Eur Spine J. 2016;25(4): 1251-1257. doi:10.1007/s00586-015-4255-9

17. Van Daele UF, Hagman F, Truijen S, Vorlat P, Van Gheluwe B, Vaes P. Differences in balance strategies between nonspecific chronic low back pain patients and healthy control subjects during unstable sitting. Spine. 2009;34(11): 1233-1238. doi:10.1097/BRS.0b013e31819 ca3ee

18. Willigenburg NW, Kingmaa I, Hoozemansab MJM, van Dieën JH. Precision control of trunk movement in low back pain patients. Hum Mov Sci. 2013;32(1):228-239. PubMed ID: 23427936 doi:10.1016/j. humov.2012.12.007

19. Berenshteyn Y, Gibson K, Hackett GC, Trem AB, Wilhelm M. Is standing balance altered in individuals with chronic low back pain? A systematic review. Disabil Rehab. 2019;41(13):1514-1523. doi:10. 1080/09638288.2018.1433240

20. da Silva RA, Vieira ER, Léonard G, et al. Age-and low back painrelated differences in trunk muscle activation during one-legged stance balance task. Gait Posture. 2019;69:25-30. doi:10.1016/j. gaitpost.2019.01.016

21. van Dieën JH, Selen LP, Cholewicki J. Trunk muscle activation in lowback pain patients, an analysis of the literature. J Electromyogr Kinesiol. 2003;13(4): 333-351. doi:10.1016/S1050-6411(03)00041-5

22. da Silva RA, Vieira ER, Cabrera M, et al. Back muscle fatigue of younger and older adults with and without chronic low back pain using two protocols: a case-control study. J Electromyogr Kinesiol. 2015;25(6): 928-936. doi:10.1016/j.jelekin.2015.10.003

23. Brumagne S, Cordo P, Verschueren S. Proprioceptive weighting changes in persons with low back pain and elderly persons during upright standing. Neurosci Lett. 2004;366(1): 63-66. PubMed ID: 15265591 doi:10.1016/j.neulet.2004.05.013

24. Appiah-Dwomoh EK, Müller S, Mayer F. Reproducibility of static and dynamic postural control measurement in adolescent athletes with back pain. Rehabil Res Pract. 2018;2018:1-8.

25. Hammami R, Behm D, Chtara M, Othman AB, Chaouach A. Comparison of static balance and the role of vision in elite athletes. J Hum Kinet. 2014;41(1):33-41. doi:10.2478/hukin-2014-0030

26. Paillard, T, Noe F, Riviere T, Marion V, Montoya R, Dupui P. Postural performance and strategy in the unipedal stance of soccer players at different levels of competition. J Athl Train. 2006; 41(2): 172176 . 
27. Shirabe NA, da Silva RA, Oliveira MR, et al. Taekwondo athletes have better postural control than Handball and American Football athletes. Revista Brasileira de Medicina do Esporte. 2017;23(6)473476. doi:10.1590/1517-869220172306170049

28. Kim HY, Kim KJ, Yang DS, Jeung SW, Choi HG, Choi WS. Screwhome movement of the tibiofemoral joint during normal gait: threedimensional analysis. Clin Orthop Surg. 2015;7(3):303-309. PubMed ID: 26330951 doi:10.4055/cios.2015.7.3.303

29. Pereira HM, Nowotny AH, Santos AB, Cardoso JR. Electromyographic activity of knee stabilizer muscles during six different balance board stimuli after anterior cruciate ligament surgery. Electromyogr Clin Neurophysiol. 2009;49(2-3);117-124. PubMed ID: 19400407

30. Grindem H, Eitzen I, Engebretsen L, Snyder-Mackler L, Risberg MA. Nonsurgical or surgical treatment of ACL injuries: knee function, sports participation, and knee reinjury. The Delaware-Oslo ACL Cohort Study. J Bone Joint Surg Am. 2014;96(15):1233-1241. PubMed ID: 25100769 doi:10.2106/JBJS.M.01054

31. Davlin CD. Dynamic balance in high level athletes. Percept Mot Skills. 2004; 98(3):1171-1176. PubMed ID: 15291203 doi:10.2466/ PMS.98.3.1171-1176

32. Nowotny AH, Calderon MG, de Souza PA, et al. Lumbar stabilization exercises versus back endurance-resistance exercise training in athletes with chronic low back pain: protocol of a randomized controlled trial. BMJ Open Sport Exerc Med. 2018;4(1):e000452. PubMed ID: 30555717 doi:10.1136/bmjsem-2018-000452

33. Cohen J. Chapter 11: Some issues in power analysis. In: Statistical Power Analysis for the Behavioral Sciences. 2nd ed. Hillsdale, NJ: Lawrence Erlbaum Associates; 1988:531-537.

34. Hosmer Jr DW, Lemeshow S, Sturdivant RX. Special topics: Exact methods for logistic regression models, Chapter 10 (10.3). In: Applied Logistic Regression. 3rd ed. Hoboken, NJ: John Willey \& Sons; 2013:387-394.

35. Borujeni BG, Yalfani A. Reduction of postural sway in athletes with chronic low back pain through eight weeks of inspiratory muscle training: a randomized controlled trial. Clin Biomech. 2019;69:215220. doi:10.1016/j.clinbiomech.2019.09.006

36. Mueller S, Mueller J, Stoll J, Cassel M, Hirschmüller A, Mayer F. Back pain in adolescent athletes: results of a biomechanical screening. Sports Med Int Open. 2017;1(1):E16-E22. PubMed ID: 30539081 doi:10.1055/s-0042-122713

37. Radebold A, Cholewicki J, Polzhofer GK, Greene HS. Impaired postural control of the lumbar spine is associated with delayed muscle response times in patients with chronic idiopathic low back pain. Spine. 2001;26(7): 724-730. doi:10.1097/00007632-200104010-00004

38. Mok NW, Brauer SG, Hodges PW. Failure to use movement in postural strategies leads to increased spinal displacement in low back pain. Spine. 2007;32(19): E537-E543. doi:10.1097/BRS.0b013e31814541a2

39. Catalá MM, Schroll A, Laube G, Arampatzis A. Muscle strength and neuromuscular control in low-back pain: elite athletes versus general population. Front Neurosci. 2018;12:436. doi:10.3389/fnins.2018. 00436

40. Abdelraouf OR, Abdel-Aziem AA. The relationship between core endurance and back dysfunction in collegiate male athletes with and without nosspecific low back pain. Int J Sports Phys Ther. 2016; 11(3):337-344. PubMed ID: 27274419

41. Grosdent S, Demoulin C, Rodriguez de La Cruz C, et al. Lumbopelvic motor control and low back pain in elite soccer players: a crosssectional study. J Sports Sci. 2016;34(11):1021-1029. PubMed ID: 26407007 doi:10.1080/02640414.2015.1085077

42. Shenoy S, Balachander H, Sandhu JS. Long latency reflex response of superficial trunk musculature in athletes with chronic low back pain. $J$ Back Musculoskelet Rehabil. 2013;26(4):445-450. PubMed ID: 23948831 doi:10.3233/BMR-130404

43. Sung W, Abraham M, Plastaras C, Silfies SP. Trunk motor control deficits in acute and subacute low-back pain are not associated with pain or fear of movement. Spine J. 2015;15(8):1772-1782. PubMed ID: 25862508 doi:10.1016/j.spinee.2015.04.010

44. Crombez G, Vervaet L, Lysens R, Baeyens F, Eelen P. Avoidance and confrontation of painful, back-straining movements in chronic back pain patients. Behav Modif. 1998;22(1):62-77. PubMed ID: 9567737 doi:10.1177/01454455980221004

45. Pereira HM, de Campos TF, Santos MB, Cardoso JR, Garcia Mde C, Cohen M. Influence of knee position on the postural stability index registered by the biodex stability system. Gait Posture. 2008; 28(4):668-672. PubMed ID: 18573663 doi:10.1016/j.gaitpost.2008. 05.003

46. Alonso AC, Mochizuki L, Luna NM, et al. Men and women do not have the same relation between body composition and postural sway. J Morphol Sci. 2015;32(02):93-97. doi:10.4322/jms.092715

47. Luoto BM, Aalto H, Taimela S, Hurri H, Pyykkö I, Alaranta H. One footed and externally disturbed two-footed postural control in patients with chronic low back pain and healthy control subjects. Spine. 1998;23(19):2081-2089. doi:10.1097/00007632-19981001000008 